PROCEEDINGS OF THE

AMERICAN MATHEMATICAL SOCIETY

Volume 130, Number 4, Pages 1211-1219

S 0002-9939(01)06227-X

Article electronically published on August 29, 2001

\title{
SCHUR ORTHOGONALITY RELATIONS AND INVARIANT SESQUILINEAR FORMS
}

ROBERT W. DONLEY, JR.

(Communicated by Rebecca Herb)

\begin{abstract}
Important connections between the representation theory of a compact group $G$ and $L^{2}(G)$ are summarized by the Schur orthogonality relations. The first part of this work is to generalize these relations to all finite-dimensional representations of a connected semisimple Lie group $G$. The second part establishes a general framework in the case of unitary representations $(\pi, V)$ of a separable locally compact group. The key step is to identify the matrix coefficient space with a dense subset of the Hilbert-Schmidt endomorphisms on $V$.
\end{abstract}

\section{INTRODUCTION}

For a connected compact Lie group $G$, the Peter-Weyl Theorem [PW] provides an explicit decomposition of $L^{2}(G)$ in terms of the representation theory of $G$. Each irreducible representation occurs in $L^{2}(G)$ with multiplicity equal to its dimension. The set of all irreducible representations are parametrized in the Theorem of the Highest Weight.

To convert representations into functions, one forms the set of matrix coefficients. To undo this correspondence, the Schur orthogonality relations express the $L^{2}$-inner product in terms of the unitary structure of the irreducible representations.

Theorem 0.1 (Schur). Fix irreducible unitary representations $\left(\pi, V^{\pi}\right),\left(\pi^{\prime}, V^{\pi^{\prime}}\right)$ of $G$. Then

$$
\int_{G}\langle\pi(g) u, v\rangle \overline{\left\langle\pi^{\prime}(g) u^{\prime}, v^{\prime}\right\rangle} d g= \begin{cases}\frac{1}{d_{\pi}}\left\langle u, u^{\prime}\right\rangle \overline{\left\langle v, v^{\prime}\right\rangle} & \text { if } \pi \cong \pi^{\prime} \\ 0 & \text { otherwise, }\end{cases}
$$

where $u, v$ are in $V^{\pi}, u^{\prime}, v^{\prime}$ are in $V^{\pi^{\prime}}, d g$ is normalized Haar measure, and $d_{\pi}$ is the dimension of $V^{\pi}$.

With a choice of orthonormal basis for each $V^{\pi}$, the orthogonality relations provide an orthonormal set in $L^{2}(G)$, and the Peter-Weyl Theorem states that this set forms a basis.

For unimodular locally compact groups, an analog of Theorem 0.1 holds for irreducible unitary representations with a square-integrable matrix coefficient [Go],

Received by the editors September 25, 2000.

2000 Mathematics Subject Classification. Primary 22D10, 22E46.

This work was partially supported by NSF grant DMS-9627447.

(C)2001 American Mathematical Society 
Di]. In this case, $d_{\pi}$ is the formal degree. These relations were discovered earlier for $S L(2, \mathbb{R})$ in $\mathrm{Ba}$.

For semisimple Lie groups, orthogonality relations for certain classes of irreducible tempered representations are known [M1]-[M3. One considers representations of $G$ that possess matrix coefficients in Hilbert spaces of functions other than $L^{2}(G)$.

Let $G$ be a separable locally compact group, and let $(\pi, V)$ be a continuous unitary representation. When $\pi$ is irreducible, the span of the matrix coefficients of $\pi$ can be identified with a dense subset of the Hilbert-Schmidt endomorphisms on $V$. The latter space admits both the structure of a continuous unitary representation of $G \times G$ and the structure of a Hilbert algebra. Thus a completion of the matrix coefficient space should reflect these properties. The orthogonality relations exhibit an analytic interpretation of the natural inner product on the space of HilbertSchmidt endomorphisms.

Apart from their intrinsic interest, orthogonality relations play a wider role in the representation theory of semisimple Lie groups [Do]. Such relations provide a direct connection between certain irreducible unitary representations and their geometric realizations as spaces of harmonic forms. The Borel-Weil-Bott Theorem concerns the simplest type of this construction, and ultimately the Langlands conjecture for discrete series representations may be recast for certain discrete series limits.

Schur orthogonality relations also occur in the theories of quantum groups, Hilbert algebras, special functions, and wavelet analysis. We leave it to the reader to pursue the relevant literature.

In section 1, a proof of Theorem 0.1 is given that allows generalizations without the invariant measure. Before stating such generalizations, results about invariant forms for finite-dimensional representations of connected semisimple Lie groups are established in section 2. Section 3 exhibits orthogonality relations in this case. The main results are Theorems 3.1, 3.4, and 3.6; these theorems generalize Theorem 0.1 in the case of finite-dimensional representations of a connected semisimple Lie group.

One desires a similar theory for irreducible unitary representations of locally compact groups. A general framework is established for this case in section 4 . The main result is Theorem 4.5; injectivity of the matrix coefficient map is asserted.

\section{The COMPACT CASE}

Let $G$ be a compact group. We recast the orthogonality relations for irreducible unitary representations in a manner that generalizes to arbitrary groups. The key step is to remove the invariant measure from the proof of Theorem 0.1; its presence is necessary only to define an invariant inner product. We recall the original proof when $\pi \cong \pi^{\prime}$. The main reference $[\mathrm{Sc}$ contains the case of finite $G$; using Haar measure, this argument generalizes for compact $G$. See, for instance, Chapter I of [K1].

First proof of Theorem 0.1. Suppose $\pi \cong \pi^{\prime}$. Let $l$ be an element of $\operatorname{End}(V)$, and define

$$
L=\int_{G} \pi(g) l \pi(g)^{-1} d g .
$$


Since $\pi(g) L=L \pi(g)$ for all $g$ in $G$, Schur's Lemma implies that $L=\lambda I$ for some $\lambda \in \mathbb{C}$. Taking traces of both sides, one has

and a substitution yields

$$
\lambda=\frac{\operatorname{tr} l}{\operatorname{dim} V},
$$

$$
\left\langle L v^{\prime}, v\right\rangle=\frac{\operatorname{tr} l}{\operatorname{dim} V} \overline{\left\langle v, v^{\prime}\right\rangle}
$$

Letting $l(w)=\left\langle w, u^{\prime}\right\rangle u$, the result follows.

Let $\left(\pi_{\lambda}, V_{\lambda},\langle\cdot, \cdot\rangle_{\lambda}\right)$ range over a complete set of representatives from each equivalence class of irreducible unitary representations of $G$ over $\mathbb{C}$. By the Peter-Weyl theorem, one has the Hilbert space decomposition

$$
L^{2}(G) \cong \bigoplus_{\lambda} V_{\lambda} \otimes V_{\lambda}^{*}
$$

The process that carries the right side to the left side is one that converts each irreducible unitary representation of $G$ into the span of its matrix coefficients. An alternative proof of Theorem 0.1 follows by comparing the natural actions of $G \times G$ in (1.1). This proof yields the relation up to a nonzero constant.

Second proof of Theorem 0.1 . Suppose $\pi \cong \pi^{\prime}$. The matrix coefficient map

$$
\phi^{\lambda}: V_{\lambda} \otimes V_{\lambda}^{*} \rightarrow L^{2}(G)
$$

is defined by linearly extending

$$
\phi_{u, v}^{\lambda}(g)=\left\langle\pi_{\lambda}(g) u, v\right\rangle_{\lambda} .
$$

When the summand is clear, we omit the label $\lambda$ from superscripts and subscripts.

Fix $\lambda$. The representation $\left(\pi \otimes \pi^{*}, V \otimes V^{*}\right)$ is irreducible with respect to $G \times G$. The map $\phi$ intertwines this action with the usual action $R \otimes L$ of $G \times G$ on $L^{2}(G)$. Since $\phi$ is nonzero, $(R \otimes L, \operatorname{Im} \phi)$ is irreducible as a representation of $G \times G$.

Define an invariant inner product on $V_{i} \otimes V_{i}^{*}$ by sesquilinearly extending

$$
\left\langle u \otimes\langle\cdot, v\rangle, u^{\prime} \otimes\left\langle\cdot, v^{\prime}\right\rangle\right\rangle_{\pi}:=\left\langle u, u^{\prime}\right\rangle \overline{\left\langle v, v^{\prime}\right\rangle} .
$$

A consequence of Schur's Lemma states that any nondegenerate invariant sesquilinear form on an irreducible representation space $V$ is unique up to a scalar. Thus, up to a scalar, $\phi$ must be a unitary equivalence of representations of $G \times G$. It follows immediately that

$$
\left\langle\phi_{u, v}, \phi_{u^{\prime}, v^{\prime}}\right\rangle_{L^{2}(G)}=c\left\langle u, u^{\prime}\right\rangle \overline{\left\langle v, v^{\prime}\right\rangle}
$$

where $c$ is a nonvanishing constant.

In general, we wish to consider variations of Theorem 0.1 that do not depend on square-integrability. The product of two matrix coefficients lies in $L^{1}(G)$. For $f$ in $L^{1}(G)$, define $T f=\int_{G} f(g) d g$. One has

$$
T\left((R \otimes L)\left(g_{1}, g_{2}\right) f\right)=\int_{G} f\left(g_{2}^{-1} g g_{1}\right) d g=T f .
$$

Thus $T$ interwines $R \otimes L$ with the trivial representation of $G \times G$. 
For generalizations of Theorem 0.1, the key step is to find a nonzero bi-invariant linear functional defined on

$$
\begin{aligned}
\operatorname{Im} \phi \otimes \overline{\operatorname{Im} \phi} & \cong\left(V \otimes V^{*}\right) \otimes \overline{\left(V \otimes V^{*}\right)} \\
& \cong\left(V \otimes V^{*}\right) \otimes\left(V \otimes V^{*}\right)^{*} .
\end{aligned}
$$

In section 3, we apply this approach to finite-dimensional representations of connected semisimple Lie groups; certain derivatives of the delta function replace integration as the functional $T$.

\section{INVARIANT SESQUILINEAR FORMS}

Let $G$ be a connected semisimple Lie group with finite center. If $G$ is simple and noncompact, there are no nontrivial irreducible unitary representations of finite dimension. Adapting the second proof in section 1, one can formulate a variation of Theorem 0.1 when $V$ admits a nondegenerate invariant sesquilinear form. Since not all irreducible finite-dimensional representations admit such forms, two cases arise.

Proposition 2.1. Let $(\pi, V)$ be an irreducible finite-dimensional representation of $G$. $V$ admits a nondegenerate invariant sesquilinear form if and only if $V$ is equivalent to $\overline{V^{*}}$, the complex conjugate of the dual representation of $V$. This form is uniquely determined up to a scalar.

If $(\pi, V)$ does not admit such a form, define $V_{\theta}=V \oplus \overline{V^{*}}$. Fix constants $c_{1}$ and $c_{2}$. If both $c_{1}$ and $c_{2}$ are nonzero, then

$$
\left\langle(u, v),\left(u^{\prime}, v^{\prime}\right)\right\rangle=c_{1} v\left(u^{\prime}\right)+c_{2} \overline{v^{\prime}(u)}
$$

defines a nondegenerate invariant sesquilinear form on $V_{\theta}$, and all such forms arise in this manner.

One can distinguish between the two cases of Proposition 2.1 using highest weight theory. A concise statement follows if we choose a positive root system according to Chapter VI.8 of [K2]. These special systems are used in the construction of Vogan diagrams.

Let $\mathfrak{g}_{0}$ denote the Lie algebra of $G$. Real Lie algebras are denoted by a "0" subscript; for the complexification, we delete this subscript. We extend representations of $\mathfrak{g}_{0}$ to $\mathfrak{g}$ complex-linearly.

Fix a maximally compact subgroup $K$ of $G$, let $\mathfrak{g}_{0}=\mathfrak{k}_{0} \oplus \mathfrak{p}_{0}$ be the associated Cartan decomposition, and let $\theta$ be the associated Cartan involution. Let $\mathfrak{h}_{0}=$ $\mathfrak{t}_{0} \oplus \mathfrak{a}_{0}$ be a $\theta$-stable fundamental Cartan subalgebra of $\mathfrak{g}_{0}$; that is, $\mathfrak{h}_{0}$ is chosen so that $\mathfrak{t}_{0}$ is a Cartan subalgebra of $\mathfrak{k}_{0}$. Weights of finite-dimensional representations take real values on $\mathfrak{h}_{\mathbb{R}}=i \mathfrak{t}_{0}+\mathfrak{a}_{0}$.

Form the set of roots $\Delta(\mathfrak{g}, \mathfrak{h})$. Then no root vanishes on $\mathfrak{t}$. We choose a positive system $\Delta^{+}$that is invariant under the action of $\theta$. Since no root vanishes on $\mathfrak{t}$, one can use a lexicographic ordering of $\mathfrak{h}$ built by following a basis of $i \mathfrak{t}_{0}$ with a basis of $\mathfrak{a}_{0}$. The set of simple roots $\Sigma(\mathfrak{g}, \mathfrak{h})$ is also $\theta$-stable.

In the irreducible case, existence of a nondegenerate invariant sesquilinear form is equivalent to the condition that the set of weights be closed under the map $\lambda \mapsto \theta \lambda=-\bar{\lambda}$. Since the fundamental Weyl chamber is $\theta$-invariant, it is sufficient to check that the highest weight is fixed by this map. Thus one has the following well-known result: 
Proposition 2.3. Form $\Delta^{+}(\mathfrak{g}, \mathfrak{h})$ as above. The irreducible finite-dimensional representation $(\pi, V)$ of $G$ admits a nondegenerate invariant sesquilinear form if and only if the highest weight $\lambda_{0}$ vanishes on $\mathfrak{a}$.

Specifically:

(1) if rank $G=\operatorname{rank} K$, then all irreducible representations admit such forms, and

(2) when $\operatorname{rank} G>\operatorname{rank} K$, let

$$
\lambda_{0}=\sum_{\alpha \in \Sigma} m_{\alpha} \lambda_{\alpha}
$$

where $\lambda_{\alpha}$ is the fundamental weight corresponding to $\alpha$. Then $V$ admits such a form if and only if $m_{\alpha}=m_{\theta \alpha}$ for all $\alpha$ in $\Sigma(\mathfrak{g}, \mathfrak{h})$.

The proposition gives no indication of an explicit realization when such a form exists. Often these forms arise naturally. For instance, when $G=S U(p, q)$, all irreducible representations occur as summands in the tensor algebra of the standard representation. It is not difficult to identify summands for which the induced forms are nondegenerate.

For an irreducible admissible representation, the existence of an invariant sesquilinear form depends on its Langlands' parameters, as seen in Theorem 16.6 of [K1].

\section{The general Finite Dimensional CASe}

Let $G$ be a connected semisimple Lie group with finite center. We consider certain derivatives of the delta function as a replacement for integration in the orthogonality relations. If $G$ is also compact, one obtains an additional expression for such relations.

Assume that $(\pi, V)$ is irreducible with nondegenerate invariant sesquilinear form $\langle\cdot, \cdot\rangle$. Let $V_{1}, \ldots, V_{k}$ denote the nontrivial irreducible representation spaces of $G$ for which the highest weights occur in the convex hull of the weight set of $V \otimes V^{*}$. For each $V_{i}$, choose an element $\Omega_{i}$ in $Z(\mathfrak{g})$ that annihilates the trivial representation but does not annihilate $V_{i}$; then $\Omega_{i}$ acts on $V_{i}$ by the nonzero scalar $c_{i}$. Define an element of $Z(\mathfrak{g})$ by

$$
D_{V}=\prod_{i=1}^{k}\left(\Omega_{i}-c_{i}\right)
$$

Identifying $D_{V}$ with a bi-invariant differential operator, we have

Theorem 3.1. Let $(\pi, V)$ be an irreducible finite-dimensional representation of $G$ that admits a nondegenerate invariant sesquilinear form $\langle\cdot, \cdot\rangle$. For some fixed constant $c_{V}$ and all $u, u^{\prime}, v, v^{\prime}$ in $V$,

$$
\begin{aligned}
\left\langle\left\langle\phi_{u, v}, \phi_{u^{\prime}, v^{\prime}}\right\rangle\right\rangle_{V} & :=R\left(D_{V}\right)\left(\phi_{u, v} \cdot \overline{\phi_{u^{\prime}, v^{\prime}}}\right) \\
& =c_{V}\left\langle u, u^{\prime}\right\rangle \overline{\left\langle v, v^{\prime}\right\rangle} .
\end{aligned}
$$

This follows immediately since $D_{V}$ annihilates all constituents except the trivial $G \times G$-type (the constant functions) in (1.6); no other constituent has a trivial factor.

We give an example of specific $\Omega_{i}$. Assume we have fixed a positive root system with respect to a $\theta$-stable Cartan subalgebra. Let $\delta$ denote half the sum of the positive roots. A distinguished element of $Z(\mathfrak{g})$ is given by the Casimir operator 
$\Omega$. If we choose a basis $\left\{X_{1}, \ldots, X_{m}\right\}$ for $\mathfrak{g}$ and let $\left\{X^{1}, \ldots, X^{m}\right\}$ be the dual basis for $\mathfrak{g}$ with respect to the Killing form $\langle\cdot, \cdot\rangle$, then

$$
\Omega=\sum_{i=1}^{m} X_{i} \cdot X^{i}
$$

as an element of $U(\mathfrak{g})$. The definition is independent of the basis chosen. This element acts on the irreducible representation with highest weight $\lambda$ by

$$
c_{\lambda}=\langle\lambda+\delta, \lambda+\delta\rangle-\langle\delta, \delta\rangle
$$

This scalar is nonzero if and only if $\lambda \neq 0$.

Note that (3.2) holds when $D_{V}$ is multiplied by an element of $U(\mathfrak{g})$ that does not annihilate the trivial representation.

Consider the case when $V$ is irreducible and has no nonzero invariant sesquilinear form. Form an operator $D_{V}$ as above with respect to $V_{\theta}$. Choose a nondegenerate invariant form $\langle\cdot, \cdot\rangle$ on $V_{\theta}$ as in (2.2). Since $\operatorname{Ker} \phi$ is nontrivial, a correct formulation of the orthogonality relation must have a restricted domain. Because finite-dimensional representations of $G \times G$ are fully reducible, Ker $\phi$ has an invariant complement $H_{\pi}$ in $V_{\theta} \otimes V_{\theta}^{*}$. Since $V$ and $\overline{V^{*}}$ are inequivalent, $H_{\pi}$ has two inequivalent irreducible constituents. Hence

Theorem 3.4. Let $(\pi, V)$ be an irreducible finite-dimensional representation of $G$ that admits no nondegenerate invariant sesquilinear form. Suppose the matrix coefficients of $V_{\theta}$ are constructed with respect to $\langle\cdot, \cdot\rangle$. For all $S, T$ in $H_{\pi}$,

$$
\begin{aligned}
\left\langle\left\langle\phi_{S}, \phi_{T}\right\rangle\right\rangle_{V} & :=R\left(D_{V}\right)\left(\phi_{S} \cdot \overline{\phi_{T}}\right) \\
& =\langle S, T\rangle_{\pi}
\end{aligned}
$$

where $\langle\cdot, \cdot\rangle_{\pi}$ is an invariant sesquilinear form on $H_{\pi}$ that depends only on $D_{V}$ and $\langle\cdot, \cdot\rangle$. We may assume $\langle\cdot, \cdot\rangle_{\pi}$ descends from an invariant form on $V_{\theta} \otimes V_{\theta}^{*}$. That is, there exist $\langle\cdot, \cdot\rangle_{i}(i=1,2)$ as in $(2.2)$ such that

$$
\left\langle u \otimes\langle\cdot, v\rangle, u^{\prime} \otimes\left\langle\cdot, v^{\prime}\right\rangle\right\rangle_{\pi}=\left\langle u, u^{\prime}\right\rangle_{1} \overline{\left\langle v, v^{\prime}\right\rangle_{2}}
$$

for all $u, u^{\prime}, v, v^{\prime}$ in $V_{\theta}$.

Let $\left(\pi_{U}, U\right)$ and $\left(\pi_{W}, W\right)$ be inequivalent irreducible representations of $G$ that are not summands of the same $V_{\theta}$. If $\pi_{U}$ (resp. $\pi_{W}$ ) does not admit a nondegenerate invariant sesquilinear form, substitute $U$ (resp. $W$ ) with $U_{\theta}$ (resp. $W_{\theta}$ ). By Schur's lemma, $\operatorname{Hom}_{G}(U, W)=0$.

Since no trivial type occurs, it follows that

Theorem 3.6. Let $\left(\pi_{U}, U\right)$ and $\left(\pi_{W}, W\right)$ be finite-dimensional representations of $G$ with nondegenerate invariant sesquilinear forms. If $\pi_{U}$ and $\pi_{W}$ have no equivalent summands, then there exists a finite-dimensional representation $\left(\pi_{V}, V\right)$ with such a form and with associated element $D_{V}$ such that

$$
\begin{aligned}
\left\langle\left\langle\phi_{u, v}, \phi_{u^{\prime}, v^{\prime}}\right\rangle\right\rangle_{V} & :=R\left(D_{V}\right)\left(\phi_{u, v} \cdot \overline{\phi_{u^{\prime}, v^{\prime}}}\right) \\
& =0,
\end{aligned}
$$

for all $u, v$ in $U$ and $u^{\prime}, v^{\prime}$ in $W$.

If we fix a basis for each finite-dimensional representation (irreducible with nondegenerate invariant sesquilinear form, or of type $V_{\theta}$ ), linear independence of any finite set of matrix coefficients formed from these bases follows. 


\section{THE GENERAL UNITARY CASE}

Let $G$ be a separable locally compact group, let $V$ be a separable complex Hilbert space, and suppose $(\pi, V,\langle\cdot, \cdot\rangle)$ is a continuous unitary representation of $G$. When $\pi$ is irreducible, we identify the span of the matrix coefficients of $\pi$ with a dense subspace of $\mathcal{H} S O(V)$, the space of Hilbert-Schmidt endomorphisms on $V$. From this subspace, the coefficient space inherits a pre-Hilbert space structure that is $G \times G$-invariant. The nonvanishing orthogonality relation is the expression of the Hilbert-Schmidt norm in terms of analytic operators.

Since $(\pi, V)$ is a continuous representation, every matrix coefficient lies in $C_{b}(G)$, the space of bounded continuous functions on $G$. Let $V \otimes V^{*}$ be the algebraic tensor product of $V$ and $V^{*}$. Define the matrix coefficient map

$$
\phi: V \otimes V^{*} \rightarrow C_{b}(G)
$$

by linearly extending

$$
u \otimes\langle\cdot, v\rangle \mapsto \phi_{u, v},
$$

where

$$
\phi_{u, v}(g)=\langle\pi(g) u, v\rangle .
$$

With respect to the induced inner product, $V \otimes V^{*}$ is a pre-Hilbert space. We identify the Hilbert space completion of $V \otimes V^{*}$ with the space $\mathcal{H} S O(V)$ of HilbertSchmidt endomorphisms on $V$. That is, $\mathcal{H} S O(V)$ is the set of all bounded endomorphisms $S$ on $V$ such that

$$
\langle S, S\rangle_{H S}=\sum_{x \in X}\langle S x, S x\rangle<\infty,
$$

where $X$ is an orthonormal basis for $V$. This sum is independent of the choice of $X$. Furthermore $\mathcal{H} S O(V)$ is preserved by the $G \times G$-action

$$
\left(\operatorname{Hom}(\pi, \pi)\left(g_{1}, g_{2}\right)\right) S=\pi\left(g_{1}\right) S \pi\left(g_{2}\right)^{-1} .
$$

As a representation of $G \times G,\left(\operatorname{Hom}(\pi, \pi), \mathcal{H} S O(V),\langle\cdot, \cdot\rangle_{H S}\right)$ is the Hilbert tensor product of $(\pi, V)$ and $\left(\pi^{*}, V^{*}\right)$. If $\pi$ is irreducible, then $\operatorname{Hom}(\pi, \pi)$ is irreducible as a representation of $G \times G$ ([Ma], Theorem 3.1). One has

Theorem 4.5. Let $G$ and $(\pi, V,\langle\cdot, \cdot\rangle)$ be as above. If $\pi$ is irreducible, then the span of the matrix coefficients of $\pi$ admits a completion $\left(R \otimes L, \mathcal{H}_{\pi},\langle\cdot, \cdot\rangle_{\pi}\right)$ as an irreducible unitary representation of $G \times G$ with

$$
\left\langle\phi_{u, v}, \phi_{u^{\prime}, v^{\prime}}\right\rangle_{\pi}:=\left\langle u, u^{\prime}\right\rangle \overline{\left\langle v, v^{\prime}\right\rangle}
$$

for all $u, u^{\prime}, v, v^{\prime}$ in $V$.

Proof. It is enough to show that $\phi$ is injective. If we identify $V \otimes V^{*}$ with the space of finite-rank endomorphisms of $V$ that are bounded, then one has $\phi_{T}(g)=\operatorname{tr}(\pi(g) T)$ for such $T$. Suppose $T$ is in $\operatorname{Ker} \phi$. Let $\left\{v_{1}, \ldots, v_{k}\right\}$ be a basis for the range of $T$. Boundedness of $T$ implies that there are unique $w_{i}$ such that

$$
T(x)=\sum_{i}\left\langle x, w_{i}\right\rangle v_{i} .
$$

Hence $\phi_{T}(g)=\sum_{i}\left\langle\pi(g) v_{i}, w_{i}\right\rangle$. 
Irreducibility of $\pi$ implies that there exists constants $c_{1}, \ldots, c_{k}$ and group elements $g_{1}, \ldots, g_{k}$ such that $w_{i}^{\prime}=\sum_{j} c_{j} \pi\left(g_{j}\right) v_{i}$ is sufficiently close to $w_{i}$. Using the formula for $\phi_{T}$, one has

$$
\begin{aligned}
0 & =\sum_{j} c_{j} \phi_{T}\left(g_{j}\right)=\sum_{i, j} c_{j}\left\langle\pi\left(g_{j}\right) v_{i}, w_{i}\right\rangle \\
& =\sum_{i}\left\langle w_{i}^{\prime}, w_{i}\right\rangle .
\end{aligned}
$$

Since $w_{i}^{\prime}$ can be made arbitrarily close to $w_{i}$, each $w_{i}=0$, and it follows that $T=0$.

The nonvanishing orthogonality relation problem may be phrased as

Open Problem 4.7. When $\pi$ is irreducible, find a function-theoretic description of $\langle\cdot, \cdot\rangle_{\pi}$ on the image of $\phi$.

As a further example, the matrix coefficients of any character of an abelian group are scalar multiples of the character; the delta function at any point implements $\langle\cdot, \cdot\rangle_{\pi}$.

The natural approach to Open Problem 4.7 is to start with $\langle\cdot, \cdot\rangle_{\pi}$ and deduce (4.6). Consider the usual formulation for square-integrable representations of a nonunimodular locally compact group [DM]. In this case, orthogonality relations do not conform to (4.6). Let $d g$ be a left Haar measure for $G$, and consider the left regular representation on $L^{2}(G)$. The right action is bounded, but not unitary. One derives an analog of Theorem 0.1 in which the formal degree is replaced with a self-adjoint positive operator; see Theorem 3 of [DM]. For a short account of this theory using reproducing kernels, see [Ca].

We describe $\langle\cdot, \cdot\rangle_{\pi}$ for some cases where square-integrability does not apply [M2]M3. Assume $G$ is a linear connected semisimple Lie group. Fix a real cuspidal parabolic subgroup $M A N$ of $G$, let $\left(\sigma_{M}, V_{M}\right)$ be a discrete series representation of $M$, and choose imaginary $\nu$ in $\mathfrak{a}_{0}^{*}$. Suppose the corresponding unitary principal series representation $(\pi, V)$ has regular infinitesimal character. Then $\pi$ is irreducible and tempered. Set $p=\operatorname{dim} A$. Define a seminorm on $C^{\infty}(G)$ by

$$
\|f\|_{p}^{2}=\lim _{\varepsilon \rightarrow 0^{+}} \varepsilon^{p} \int_{G}|f(g)|^{2} e^{-\varepsilon d(g)} d g
$$

where $d(g)$ denotes the Riemannian distance from $g K$ to the origin in $G / K$ and $d g$ is a Haar measure. Let $H_{p}(G, \chi)$ denote the space of all $C^{\infty}$-functions $f$ on $G$ such that $\|(R \otimes L)(u, v) f\|_{p}<\infty$ for all $u, v$ in $U\left(\mathfrak{g}_{0}\right)$ and $(R(z)-\chi(z)) f=0$ for all $z$ in $Z(\mathfrak{g})$. This space is a pre-Hilbert space with respect to $\|\cdot\|_{p}$; we denote its completion by $H(G, \chi)$. Then $(\pi, V)$ is realized on $H(G, \chi)$. See Theorems I-V of [M3].

In [M1] and M2], other representations associated to the case $p=1$ are considered. These cases include limits of holomorphic discrete series representations and nonholomorphic limits with one degree of singularity.

\section{ACKNowledgments}

The author thanks David Vogan and Gregg Zuckerman for helpful conversations. Further useful comments were made by Leticia Barchini, Paul Friedman, and Roger Zierau. 


\section{REFERENCES}

[Ba] V. Bargmann, Irreducible unitary representations of the Lorentz group, Ann. of Math. 48 (1947), 568-640. MR 9:133a

[Ca] A. L. Carey, Square-integrable representations of non-unimodular groups, Bull. Austral. Math. Soc. 15 (1976), 1-12. MR 55:3153

[Di] J. Dixmier, $C^{*}$-Algebras, North-Holland Pub. Co., New York, 1977. MR 56:16388

[Do] R. W. Donley, Jr., Orthogonality relations and harmonic forms for semisimple Lie groups, J. Funct. Anal. 170 (2000), 141-160. CMP 2000:07

[DM] M. Duflo and C. C. Moore, On the regular representation of a nonunimodular locally compact group, J. Funct. Anal. 21 (1976), 209-243. MR 52:14145

[Go] R. Godement, Sur les relations d'orthogonalité de V. Bargmann, I and II, C. R. Acad. Sci. Paris 225 (1947), 521-523 and 657-659. MR 9:134a MR 9:134b

[K1] A. W. Knapp, Representation Theory of Semisimple Lie Groups, Princeton Univ. Press, Princeton, 1986. MR 87j:22022

[K2] A. W. Knapp, Lie Groups Beyond an Introduction, Birkhäuser, Boston, 1996. MR 98b:22002

[Ma] G. W. Mackey, The Theory of Unitary Group Representations, Univ. Chicago Press, Chicago, 1976. MR 53:686

[M1] H. Midorikawa, On certain irreducible representations for the real rank one classical groups, J. Fac. Sci. Univ. Tokyo 21 (1974), 435-459. MR 51:809

[M2] H. Midorikawa, Schur orthogonality relations for certain nonsquare integrable representations of real semisimple Lie groups, Tokyo J. Math. 8 (1985), 303-336. MR 87g:22017

[M3] H. Midorikawa, Schur orthogonality relations for nonsquare integrable representations of real semisimple linear group and its application, Representations of Lie Groups, Advanced Studies in Pure Mathematics, vol. 14, 257-287, Academic Press, Boston, 1988. MR 91g:22022

[PW] F. Peter and H. Weyl, Die Vollständigkeit der primitiven Darstellungen einer geschlossenen kontinuierlichen Gruppe, Math. Annalen 97 737-755.

[Sc] I. Schur, Neue Begründung der Theorie der Gruppencharaktere, Sitzungsber. Preuss. Akad., 1905, 406-432.

Department of Mathematics, University of North Texas, Denton, Texas 76203

E-mail address: rdonley@unt.edu 